MAP kinase activation was also reduced in these spinal slices.

To demonstrate the potential of Homer1a as a therapeutic target, the investigators generated Homer1a-overexpressing mice. These mice showed reduced thermal hyperalgesia associated with peripheral hindpaw inflammation, but other nociceptive behaviors and motor performance were unaffected. The authors conclude that the activity-dependent remodeling of glutamatergic synapses, particularly through Homer1a manipulation, is a novel but feasible therapeutic approach for the treatment of inflammatory pain.

Original article Tappe A et al. (2006) Synaptic scaffolding protein Homer la protects against chronic inflammatory pain. Nat Med 12: 677-681

\section{Reduced levels of DHEA linked with fatigue in multiple sclerosis}

Patients with multiple sclerosis (MS) commonly experience symptoms of fatigue, but the etiology of this symptom is unclear. Studies in patients with non-neurological diseases who experience fatigue have indicated a role for circulating steroids, and a new study conducted in MS patients has found that decreased levels of dehydroepiandrosterone (DHEA) and DHEA sulfate (DHEAS) are associated with fatigue.

Téllez and colleagues studied 73 patients with progressive MS who had been recruited to a trial in which they randomly received either interferon- $\beta 1 \mathrm{~b}$ or placebo for 24 months. Using the Fatigue Severity Scale (FSS), the researchers assessed patients' fatigue levels before treatment, and 3, 6, 12 and 24 months after commencement of treatment; blood samples were also collected at these time points. Patients who experienced fatigue (an FSS score $>5$ ) throughout the entire study period (sustained fatigue; $n=29$ ) and those who were fatigue-free for the whole study $(n=9)$ were included in the statistical analysis. As depression is thought to be related to fatigue in MS, analysis was adjusted to take into account patients' levels of depression.

At all time points, there was no significant difference in FSS scores or serum levels of DHEA and DHEAS between patients receiving interferon- $\beta 1 \mathrm{~b}$ and those on placebo. The mean DHEA and DHEAS levels at all time points were lower in patients with sustained fatigue than in those who were fatigue-free.
The authors conclude that serum levels of DHEA or DHEAS can be used as a marker of fatigue in patients with MS, and that DHEA replacement therapy could form a new treatment option for affected patients.

Original article Téllez N et al. (2006) Fatigue in progressive multiple sclerosis is associated with low levels of dehydroepiandrosterone. Mult Scler 12: 487-494

\section{Can stereotactic radiosurgery alone be used for the treatment of brain metastases?}

Researchers from Japan have reported that treatment with whole-brain radiotherapy (WBRT) plus stereotactic radiosurgery (SRS) confers an increase in brain tumor control without evidence of survival advantage in patients with brain metastases, when compared with SRS alone. This is the first prospective, randomized, multi-institutional study to compare the two treatment regimens.

Patients with 1-4 brain metastases, each $3 \mathrm{~cm}$ or less in diameter, were randomized to WBRT plus SRS $(n=65)$ or SRS alone $(n=67)$. The dose of WBRT was $30 \mathrm{~Gy}$ given in 10 fractions over a 2-2.5-week period. For metastatic lesions $\leq 2 \mathrm{~cm}$ and $>2 \mathrm{~cm}$ in diameter, the SRS doses were 22-25 Gy and 18-20 Gy, respectively. In patients undergoing combined therapy, the SRS dose was reduced by $30 \%$.

The median survival time did not differ significantly between the two treatment groups (WBRT plus SRS 7.5 months; SRS alone 8.0 months). The brain tumor recurrence rate was, however, significantly higher in patients who received SRS alone $(P<0.001)$, and more patients in the SRS group developed new brain metastases than those in the combined therapy group $(P=0.003)$. Multivariate analyses revealed a significantly reduced risk of tumor recurrence $(P<0.001)$ in patients who underwent WBRT plus SRS.

The authors conclude that the use of WBRT plus SRS does not improve survival, but that using SRS alone for the treatment of brain metastases results in a high relapse rate, necessitating frequent assessment to monitor brain tumor recurrence.

Original article Aoyama $\mathrm{H}$ et al. (2006) Stereotactic radiosurgery plus whole-brain radiation therapy vs stereotactic radiosurgery alone for treatment of brain metastases. JAMA 295: 2483-2491 